\title{
Modificación del grosor íntima-media carotídeo según factores de riesgo clásicos y síndrome metabólico con o sin inflamación
}

\author{
Mónica Acevedo, Pilar Arnaíz, Ramón Corbalán, Iván Godoy, Daniel Morales, \\ Mónica Chalhub, Beatriz Guzmán, Carlos Navarrete, Ximena Berríos. \\ Departamentos de Enfermedades Cardiovasculares y Salud Pública, \\ Pontificia Universidad Católica de Chile, Santiago, y Departamento de Matemática, \\ Universidad del Bío Bío, Concepción
}

Recibido el 08 de junio de 2009, aceptado 21 de septiembre de 2009

Rev Chil Cardiol 2009; 28: 337-348

\section{Resumen}

El grosor íntima-media carotideo (CIMT) es un marcador de aterosclerosis subclínica y eventos isquémicos cerebrales y coronarios. Si bien los valores normales promedio en Chile, han sido publicados, no existen datos locales de cómo se modifica el CIMT según la carga de factores de riesgo (FR) cardiovascular. Objetivos: Analizar la modificación del CIMT según la carga de FR clásicos, presencia de síndrome metabólico (SMET) y SMET más inflamación.

Métodos: Hombres y mujeres de Santiago sin antecedente de eventos isquémicos previos, nivel socio económico medio, medio bajo y medio alto. En todos se realizó encuesta sobre antecedentes demográficos, FR cardiovascular, y medición de PA, IMC, cintura, y perfil lipídico, glicemia y proteína C-reactiva ultrasensible (ePCR) en ayuno. Para diagnóstico de SMET se usó NCEP ATPIII (2001), y para inflamación, ePCR> 2 mg/L (valor de corte de > riesgo por AHA). El CIMT se midió en carótida común derecha e izquierda con software MATH ${ }^{\circledR}$. Resultados: Se incluyeron en el análisis 999 sujetos (508 hombres), edad promedio $43.8 \pm 11$ años. La prevalencia de HTA fue 30\%, dislipidemia 68\%, tabaquismo 47\%, y SMET 24\%. EI CIMT medio promedio fue $0.61 \pm 0.1 \mathrm{~mm}$ y la ePCR promedio $2.22 \mathrm{mg} / \mathrm{L}$. La modificación del CIMT medio $(\mathrm{mm})$ según número de FR (edad $>45 / 55$ hombre/mujer, diabetes, HTA, colesterol total $>200, H D L<40 / 50$, tabaquismo e historia familiar), fue la siguiente: $0 F R=0.55 \pm 0.08 ; 1-2 F R=0.59 \pm 0.091 ; \geq 3 F R=0.67 \pm 0.12(p<0.0001)$. Asímismo, el CIMT aumentó según el puntaje de Framingham: $<5 \%=0.58 \pm 0.09 ; 5-10 \%=0.66 \pm 0.11 ;>10-20 \%=0.68 \pm 0.11$; $>20 \%=0.76 \pm 0.14$ ( $p<0.0001)$. El CIMT fue significativamente mayor en los sujetos con SMET, y se incrementa en forma significativa cuando se adiciona ePCR $>2 \mathrm{mg} / \mathrm{L}$. En el modelo de regresión múltiple, el CIMT se asocia en forma conjunta a Framingham $(p<0.0001)$ y SMET+ePCR $>2(p=0.01)(r 2=0.19$ para modelo). Conclusión: El CIMT aumenta según la carga de FR clásicos y en sujetos que presentan SMET. EI aumento significativo de CIMT en los sujetos con SMET e inflamación (ePCR>2), sugiere un aumento del riesgo aterosclerótico en ellos que deberá ser confirmado en estudios prospectivos con medición de eventos. Este estudio confirma la utilidad del CIMT para cuantificar carga de FR.

Palabras clave: Grosor íntima-media carotídeo, factores de riesgo cardiovascular.

Correspondencia: Dra. Mónica Acevedo B.

Departamento de Enfermedades Cardiovasculares,

Pontificia Universidad Católica de Chile

Lira 85, Primer Piso, Santiago Centro

Correo Eléctronico: macevedo@med.puc.cl 


\section{Relation of Intima-Media Thickness, traditional Risk Factors and Metabolic Syndrome in subjects with or with inflammation}

Carotid intima-media thickness (CIMT) is a marker for cerebral and coronary ischemic events. Normal values for CIMT in Chile have been published but the relation of CIMT to level of risk factors (RF) is unknown.

Aim. To analyze the relation of CIMT to level of traditional RF and the presence of metabolic syndrome (MS), with or without inflammation.

Methods. Males and females living in Santiago, Chile, with no prior history of ischemic events, of a low, medium or high socio-economic level, were studied. Demographic data, and presence of RF were obtained. Blood pressure, BMI, waist, blood lipids, blood glucose and US-CRP were measured in the fasting state. The presence of MS was defined by the NCEP-ATP III (2001) criteria. Inflammation was defined as US-CRP >2mg/L (the cutoff point for increased risk, according to the AHA). CIMT was measure in both carotid arteries using the MATH® software.

Results. 999 subjects (508 males), aged $43 \pm 11$ years old. The prevalence of hypertension (HT) was 30\%, dyslipidemia $68 \%$, tobacco use $47 \%$ and MS 24\%. Mean CIMT was $0.61 \pm 0.1 \mathrm{~mm}$ and US-PCR was $2.22 \mathrm{mg} / \mathrm{L}$. CIMT according to number of RF - age > 45/55 (male/female), diabetes, HT, total cholesterol $>200 \mathrm{mg} / \mathrm{dl}$, $H D L<40 / 50 \mathrm{mg} / \mathrm{dl}$, tobacco use and family history- were: 0 RF: $0.55 \pm 0.08 ; 1-2$ RF: $0.59 \pm 0.091 ; \geq 3$ RF: $0.67 \pm 0.12(p<0.0001)$. CIMT according to Framingham scores was: < 5\%: $0.58 \pm 0.09 ; 5-10 \%: 0.66 \pm 0.11$; $10-20 \%: 0.68 \pm 0.11$ and $>20 \%: 0.76 \pm 0.14(p<0.0001)$. CIMT was significantly greater in subjects with $M S$ and more in the group with US-PCR $>2 \mathrm{mg} / \mathrm{L}$. In multiple regression analysis, CIMT was jointly associated to Framingham score $(p<0.0001)$ and presence of $M S+U S-P C R>2 \mathrm{mg} / L(p=0.01)(r 2=0.19$ for the model). Conclusion. CIMT increases in relation to traditional RF load and the presence of MS. An increased atherosclerotic risk in subjects with MS+inflammation (US-PCR $>2 \mathrm{mg} / \mathrm{L}$ ) should be confirmed by follow up studies of cardiovascular events. This study confirms the clinical value of CIMT measurement as and indication of RF load.

Key words: Carotid intima-media thickness, cardiovascular risk factors

\section{Introducción}

El grosor íntima-media carotídeo (CIMT) es un marcador de aterosclerosis subclínica. Es medido a través de una ultrasonografía carotídea, siendo un método seguro, no invasivo y reproducible 1 . Numerosos estudios han demostrado que el CIMT se asocia a los factores de riesgo cardiovascular (CV) tradicionales ${ }^{2-5}$, y se ha postulado que la progresión del CIMT es igual al resultado de la respuesta de cada persona a su propia carga de factores de riesgo, independiente de si esta carga es leve o importante, o de su duración6. De esta manera, el CIMT da información directa de la progresión aterosclerótica a nivel de la pared arterial carotídea. Por otra parte, el CIMT se asocia, en forma independiente, a eventos CV y cerebrovasculares, como infarto al miocardio y accidente vascular encefálico isquémico, aún después de ajustar por los factores de riesgo tradicionales ${ }^{7}$.
Recientemente, Lorenz y cols. ${ }^{8}$ han confirmado esta asociación en un meta-análisis que incluyó ocho estudios y que reportó que el CIMT es un poderoso predictor de eventos vasculares.

El síndrome metabólico (SMET) se caracteriza por ser un conjunto de factores de riesgo que incluye adiposidad abdominal, hipertensión arterial, hipertrigliceridemia, colesterol HDL bajo y disglicemia9, 10. Se ha demostrado que el SMET es un predictor de diabetes mellitus tipo $2^{11}$, cardiopatía coronaria y mortalidad cardiovascular12-14. Algunos estudios han demostrado que la presencia de SMET se asocia a un aumento del CIMT15,16. Por otro lado la aterosclerosis es una enfermedad inflamatoria. Numerosos estudios epidemiológicos han demostrado que la proteína C-reactiva ultrasensible (EPCR), marcador inflamatorio, es un predictor de eventos $\mathrm{CV}$ futuros, tanto en hombres 17 como en mujeres ${ }^{18}$. Sin embargo, las opiniones han 
sido controvertidas en cuanto al poder aditivo, más allá de los factores de riesgo tradicionales, de este marcador ${ }^{19}$. Por otro lado, análisis basados en curvas de sensibilidad-especificidad, muestran que su determinación no agrega mayor información pronóstica. Pero en estudios en que la EPCR se ha agregado como factor asociado al SMET, sobretodo en mujeres, la adición de este factor inflamatorio a sujetos con SMET, determina mayor riesgo de eventos $\mathrm{CV}^{18}$. Haffner y cols. han demostrado que la EPCR elevada es un factor de riesgo potente para el desarrollo de SMET y diabetes, y han sugerido que la determinación de EPCR debiera ser considerada como otro componente del llamado SMET11, 20, 21.

En nuestro país no existen reportes de la asociación entre los factores de riesgo clásicos, puntaje de Framingham y CIMT. Dado que los valores de CIMT varían según la raza, es importante conocer si estas asociaciones se mantienen en nuestra población. Por otro lado, si bien existen algunos reportes de cómo se modifica el CIMT con la presencia o ausencia de SMET, éstos son escasos, y no existen datos publicados en la literatura de cómo cambia el CIMT en sujetos con ambos: SMET e inflamación.

El objetivo de este trabajo fue demostrar que, en sujetos chilenos, el CIMT aumenta según la agregación de factores de riesgo CV clásicos y en aquellos que tienen SMET pero, que en estos últimos, la presencia de inflamación subclínica, determina un CIMT aún mayor.

\section{Métodos}

Estudio transversal realizado en una población urbana de Santiago entre los años 2006 y 2007. La población fue seleccionada por el equipo de Salud Pública de la Universidad a través de un muestreo epidemiológico estratificado, que incluyó a adultos entre 20 y 70 años. Se incluyeron 3 estratos socioeconómicos: bajo, medio-bajo y medio-alto del sector urbano de Santiago. Se excluyeron aquellos sujetos con antecedente de enfermedad aterosclerótica conocida, así como aquellos con antecedente de infección reciente, o enfermedades inflamatorias no infecciosas conocidas.

\section{Recolección de Datos}

Los sujetos fueron entrevistados en sus hogares a través de una encuesta, que incluía preguntas sobre antecedentes demográficos, nivel de educación, antecedentes médicos previos, factores de riesgo $\mathrm{CV}$, nivel de actividad física y medicamentos actualizados. Luego, todos fueron citados al centro universitario, donde una enfermera universitaria les realizó las mediciones antropométricas, que incluyeron peso, talla, índice de masa corporal (IMC), cintura, cadera, y presión arterial sistólica y diastólica (PAS-PAD) después de 5 minutos de reposo, en 3 oportunidades (cada 3 minutos) con manguito braquial (según recomendaciones de JNC 7), y les realizó extracción de sangre venosa en ayunas, para determinación de exámenes de laboratorio: perfil lipídico, glicemia y proteína C-reactiva ultrasensible. Todos los sujetos fueron citados en una segunda oportunidad, dentro del plazo de 1 semana al centro universitario, para determinación del CIMT, oportunidad en que además fueron examinados por los médicos a cargo del estudio.

Se consideró hipertenso, a todo sujeto con diagnóstico médico de hipertensión arterial, con o sin tratamiento farmacológico y a aquellos sujetos con más de 2 determinaciones de presión arterial ${ }^{3}$ $140 / 90 \mathrm{mmHg}$. Se consideró dislipidémicos a aquellos sujetos con diagnóstico de hipercolesterolemia diagnosticada por médico, con o sin tratamiento farmacológico, y a aquellos con colesterol total $>200 \mathrm{mg} / \mathrm{dL}, \mathrm{y} / \mathrm{o}$ a aquellos con colesterol $\mathrm{HDL}<40$ en hombres $\mathrm{y}<50 \mathrm{mg} / \mathrm{L}$ en mujeres, en el examen actual. Diabetes fue definida en aquellos con diagnóstico médico de diabetes con o sin tratamiento farmacológico, y aquellos que presentaron una glicemia de ayuno mayor de $126 \mathrm{mg} / \mathrm{dL}$ en este estudio.

Toda la información fue registrada en una base de datos, especialmente diseñada para el estudio.

\section{Definición de Síndrome Metabólico y Puntaje} de Framingham

Se utilizó la definición de SMET por NCEP ATPIII22. Se definió como SMET si la persona tenía 3 o más de los siguientes criterios: a) presión arterial ${ }^{3}$ 130/85 mmHg; b) Triglicéridos 3150 mg/dL; c) colesterol $\mathrm{HDL}<40 \mathrm{y}<50 \mathrm{mg} / \mathrm{dL}$ en hombres $\mathrm{y}$ 
mujeres respectivamente; d) glicemia $3100 \mathrm{mg} / \mathrm{dL}$; e) circunferencia de cintura $>102 \mathrm{~cm}$. en hombres $\mathrm{y}>$ $88 \mathrm{~cm}$. en mujeres.

La determinación del puntaje de Framingham se realizó mediante las tablas de determinación del riesgo cardiovascular global a 10 años, y que incluyen puntaje para diabetes, publicadas por Wilson y cols en $1998^{23}$.

\section{Mediciones de laboratorio}

Las muestras para perfil lipídico, glicemia y ePCR fueron obtenidas por punción venosa, con ayuno de 12 hrs. Su medición se realizó en el laboratorio central del hospital.

a) Colesterol total, colesterol HDL y triglicéridos: Fueron medidos usando los métodos enzimáticos estándar con reactivos ad-oct (analizador Yací).

b) Colesterol LDL: Fue calculado por fórmula de Friedewald.

c) Glicemia: método glucosa oxidasa.

d) ePCR: se midió ePCR ultrasensible por método nefelométrico (Nefelómetro Dade Behring BN II; Límite de detección $0.1 \mathrm{mg} / \mathrm{L}$ ). Para evitar realizar mediciones en sujetos cursando infecciones, a todos los participantes se les recomendó diferir su evaluación, si 2 semanas previas a su citación habían cursado con algún cuadro infeccioso o inflamatorio. Se excluyeron los sujetos con ePCR > $10 \mathrm{mg} / \mathrm{L}$ (sugerente de infección aguda). Así mismo, para el análisis estadístico se usó un valor de corte de ePCR $>2 \mathrm{mg} / \mathrm{L}$, que corresponde al valor intermedio sugerido por CDC/AHA en el año 200324 como valor elevado de este marcador. Posteriormente, este valor de corte ha sido validado por estudios de eventos que han demostrado su valor predictivo.

Medición de Grosor Intima-Media Carotídeo (CIMT)

Se realizó según las recomendaciones del consenso en medición del grosor íntima-media carotídeo realizado en Manheim 200425 y actualizado el año 200626, y todas las mediciones fueron realizadas por una sola sonografista, certificada para la medición de CIMT en un estudio previo27. Para la medición del CIMT y placa se utilizó un equipo
Hewlett Packard Sonos 5500 con un transductor lineal de alta frecuencia (5-13 Mhz). La imagen se focalizó en la pared posterior de cada arteria carótida común, en áreas sin placas, dónde se eligió un segmento de $1 \mathrm{~cm}$., proximal a la bifurcación carotídea de cada lado. Se realizaron mediciones en cada carótida común en 3 sitios pre-establecidos: anterior, antero-medial y póstero-medial. Para la medición, se usó un software con detección automática de bordes (M'Ath ${ }^{\circledR}$ Std). Todas las mediciones se realizaron "off-line" y en fin de diástole. Se registró también la presencia o ausencia de placas carotídeas (protrusión en el lumen mayor al $50 \%$ del CIMT adyacente o CIMT > $1.5 \mathrm{~mm}$ ). EI valor de CIMT máximo y medio registrados correspondió al mayor valor de CIMT entre el lado derecho e izquierdo. Las mediciones se realizaron en forma ciega al resultado de los exámenes de sangre. Para efectos de análisis estadístico se utilizó sólo CIMT medio máximo. El coeficiente de variación intraoperador de nuestro grupo es de $3.8 \%$.

\section{Análisis Estadístico}

Para las Tablas 1 y 2 los resultados se expresan por media y desviación estándar. La EPCR se analizó en escala logarítmica en todas las comparaciones para corregir la asimetría de su distribución. Para la comparación de CIMT entre distintos grupos se ocupó ANOVA y regresión lineal múltiple. Las correlaciones en Tabla 3 corresponden a correlaciones parciales, interpretables como porcentaje de la varianza explicada por cada variable después de ajustar por edad y sexo. Los cálculos de Odds Ratio (OR) para CIMT elevado (Tabla 4) se basan en modelos de regresión logística, ocupando como respuesta CIMT > $0.67 \mathrm{~mm}$., correspondiente al percentil 75 de la muestra. Los gráficos en Figuras 1, 2 y 3 son de tipo Box \& Whisker, donde la línea central representa la mediana, la caja el rango intercuartil y los extremos las observaciones mínima y máxima no consideradas como "fuera de rango" (Tukey JW."Exploratory Data Analysis",1977). 
Tabla 1: Variables demográficas, prevalencia de factores de riesgo cardiovascula, medidas antropométricas, perfil lipídico, ePCR y grosor íntima-media carotideo en la población estudiada, por sexo (valores expresados como promedio \pm desviación estándar o porcentaje)

\begin{tabular}{lccc}
\hline & Total $(\mathbf{n = 9 9 9 )}$ & Hombres $(\mathbf{n = 5 0 8})$ & Mujeres (n=491) \\
\hline Edad & $43.8 \pm 10.9$ & $43.7 \pm 10.9$ & $43.9 \pm 11.0$ \\
Hipertensión Arterial \% & 30 & 32 & 27.6 \\
Dislipidemia \% & 68 & 62 & 75.1 \\
Diabetes \% & 12 & 13 & 11.6 \\
Tabaquismo \% & 47 & 48 & 45.2 \\
S. Metabólico \% & 24 & 21 & 27.7 \\
IMC (kg/m²) & $27.8 \pm 4.9$ & $27.6 \pm 3.9$ & $28.1 \pm 5.7$ \\
Colesterol Total (mg/dl) & $201.3 \pm 40.1$ & $200.1 \pm 39.7$ & $202.6 \pm 40.5$ \\
Colesterol HDL(mg/dl) & $49.5 \pm 12.5$ & $46.1 \pm 11.0$ & $53.0 \pm 13.0$ \\
Colesterol LDL(mg/dl) & $120.6 \pm 33.1$ & $119.5 \pm 33.2$ & $121.8 \pm 32.9$ \\
Triglicéridos(mg/dl) & $156.4 \pm 107.0$ & $174.3 \pm 117.1$ & $137.7 \pm 91.9$ \\
Presión Arterial Sistólica $(\mathrm{mmHg})$ & $121.2 \pm 20.6$ & $123.5 \pm 19.7$ & 118.921 .3 \\
Presión Arterial Diastólica $(\mathrm{mmHg})$ & $79.3 \pm 12.5$ & $82.0 \pm 12.1$ & $76.5 \pm 12.3$ \\
IMT medio máximo (mm) & $0.61 \pm 0.1$ & $0.62 \pm 0.1$ & $0.60 \pm 0.1$ \\
ePCR (mg/L) & $2.22 \pm 2.2$ & $1.90 \pm 1.9$ & $2.56 \pm 2.4$
\end{tabular}

Tabla 2: Grosor íntima-media carotídeo según edad y sexo. (Valores expresados en mm, como promedio \pm desviación estándar)

\begin{tabular}{lccc}
\hline Edad (años) & \multicolumn{3}{c}{ IMT (mm) } \\
\hline & Total $(\mathbf{N}=\mathbf{9 9 9})$ & Hombres (N=508) & Mujeres (N=491) \\
\hline$>20-30(n=148)$ & $0.52 \pm 0.05$ & $0.53 \pm 0.05$ & $0.51 \pm 0.05$ \\
$>30-40(n=256)$ & $0.57 \pm 0.07$ & $0.58 \pm 0.07$ & $0.55 \pm 0.06$ \\
$>40-50(n=287)$ & $0.62 \pm 0.10$ & $0.62 \pm 0.08$ & $0.62 \pm 0.11$ \\
$>50-60(n=240)$ & $0.67 \pm 0.10$ & $0.69 \pm 0.11$ & $0.65 \pm 0.09$ \\
$>60-70(n=68)$ & $0.74 \pm 0.11$ & $0.74 \pm 0.11$ & $0.74 \pm 0.11$ \\
\hline
\end{tabular}

Tabla 3: Correlaciones de Pearson (R) parciales, ajustadas por edad y sexo entre Grosor íntima-media carotídeo, Factores de Riesgo y log ePCR

\begin{tabular}{lcc}
\hline & $\mathbf{R}$ & $\mathbf{p}$ \\
\hline Edad & 0.59 & $<0.0001$ \\
IMC & 0.18 & $<0.0001$ \\
Circunferencia cintura & 0.16 & $<0.0001$ \\
Presión Arterial Sistólica & 0.28 & $<0.0001$ \\
Presión Arterial Diastólica & 0.20 & $<0.0001$ \\
Colesterol Total & 0.12 & $<0.001$ \\
Triglicéridos & 0.10 & $<0.01$ \\
Glicemia & -0.05 & $\mathrm{NS}$ \\
Colesterol LDL & 0.12 & $<0.001$ \\
Colesterol HDL & -0.11 & $<0.001$ \\
log (ePCR) & 0.25 & $<0.001$
\end{tabular}


M. Acevedo, P. Arnaíz, R. Corbalán, I. Godoy, D. Morales, M. Chalhub, et al.

Tabla 4: Riesgo de Grosor íntima-media carotídeo elevado (> al percentil 75): A) sin ajustar y B) en forma conjunta (multivariado)

A)

\begin{tabular}{lc}
\hline Variable & OR (I.C. 95\%) \\
\hline Edad & $1.14(1.12-1.16)$ \\
Puntaje de Framingham & $1.13(1.10-1.16)$ \\
Síndrome Metabólico & $2.61(2.23-3.05)$ \\
log ePCR & $1.41(1.21-1.65)$ \\
ePCR $>2 \mathrm{mg} / \mathrm{L}$ & $1.65(1.21-2.24)$ \\
S. Metabólico + ePCR>2 mg/L & $3.81(2.57-5.69)$ \\
\hline
\end{tabular}

B)

\begin{tabular}{lc}
\hline Variable & OR (I.C. 95\%) \\
\hline P. Framingham & $1.12(1.08-1.15)$ \\
S. Metabólico + ePCR $>2 \mathrm{mg} / \mathrm{L}$ & $1.76(1.10-2.82)$ \\
\hline
\end{tabular}

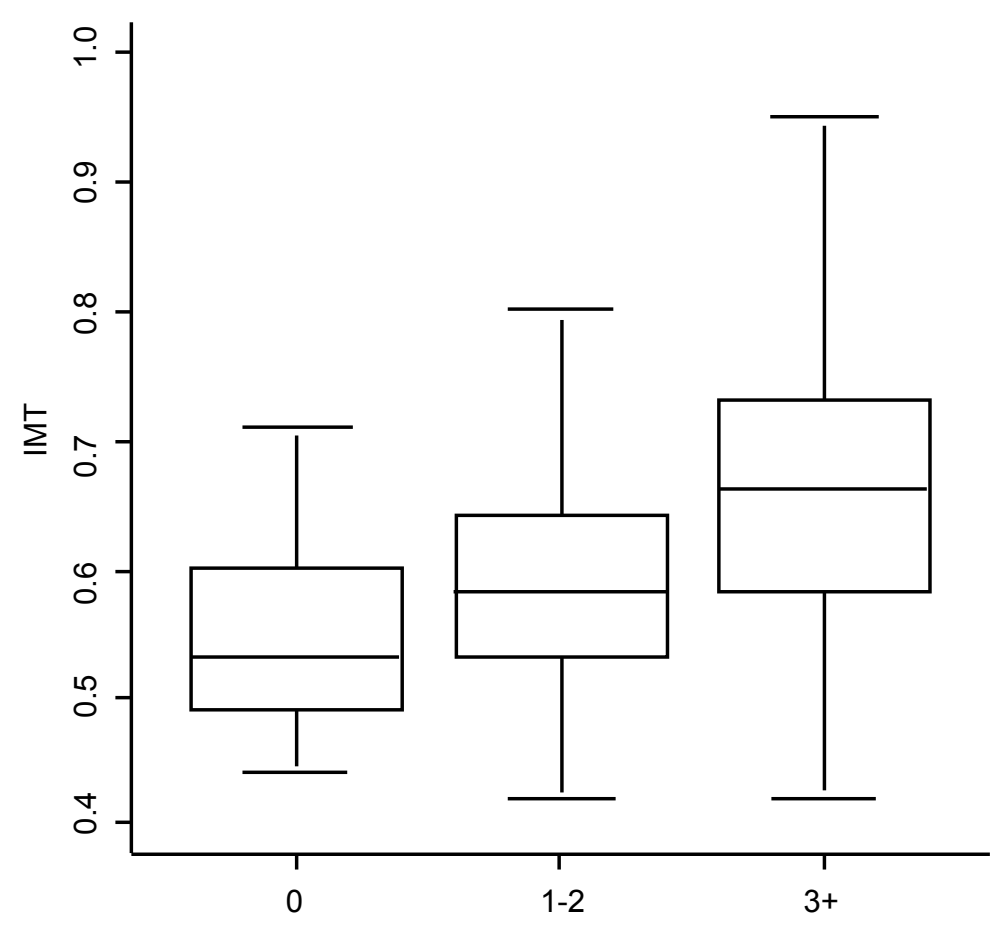

Número de Factores de Riesgo Cardiovascular

$p<0.0001$

Figura 1: Grosor Intima-Media Carotídeo según número de Factores de Riesgo Cardiovascular. (Gráfico Box \& Whisker, donde la línea central representa la mediana, la caja el rango intercuartil y los extremos las observaciones mínima y máxima). 


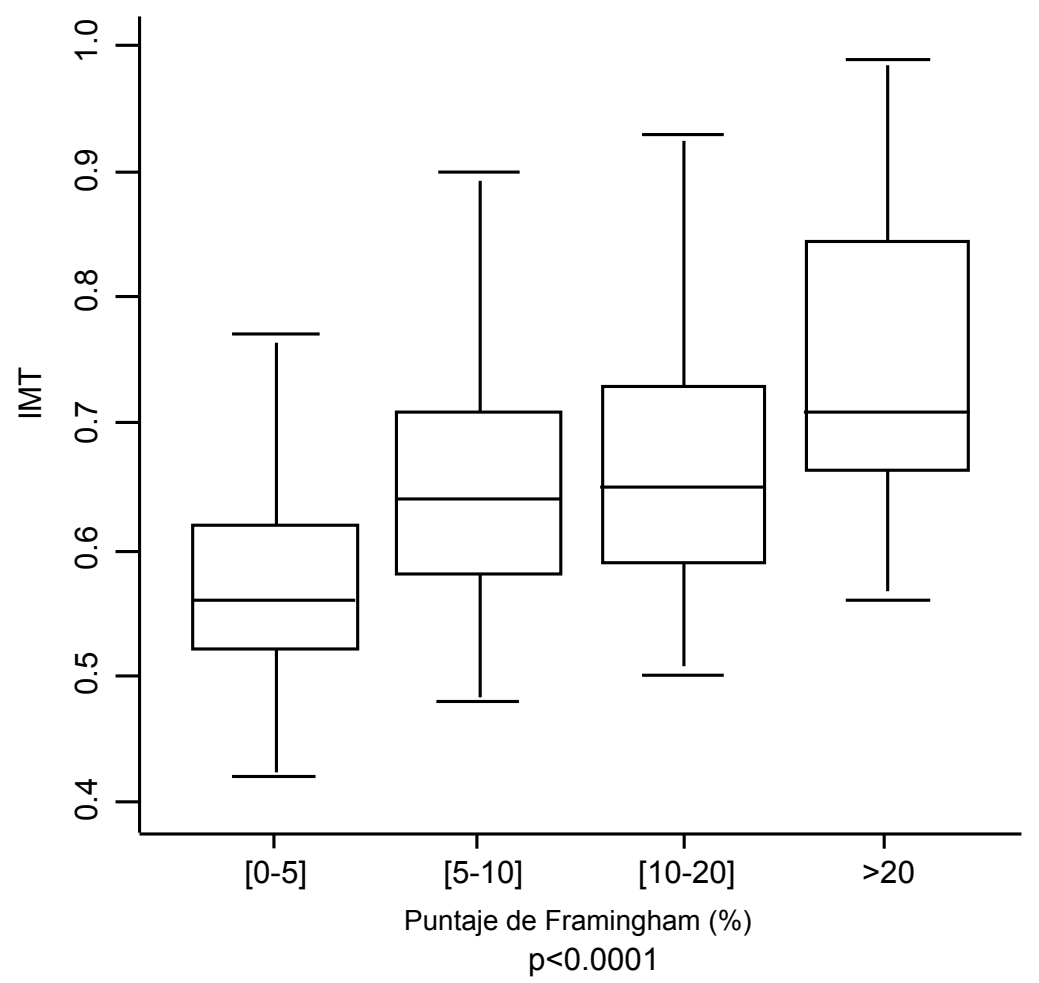

Figura 2: Grosor Intima-Media Carotídeo según Puntaje de Framingham (Gráfico Box \& Whisker, donde la línea central representa la mediana, la caja el rango intercuartil y los extremos las observaciones mínima y máxima)

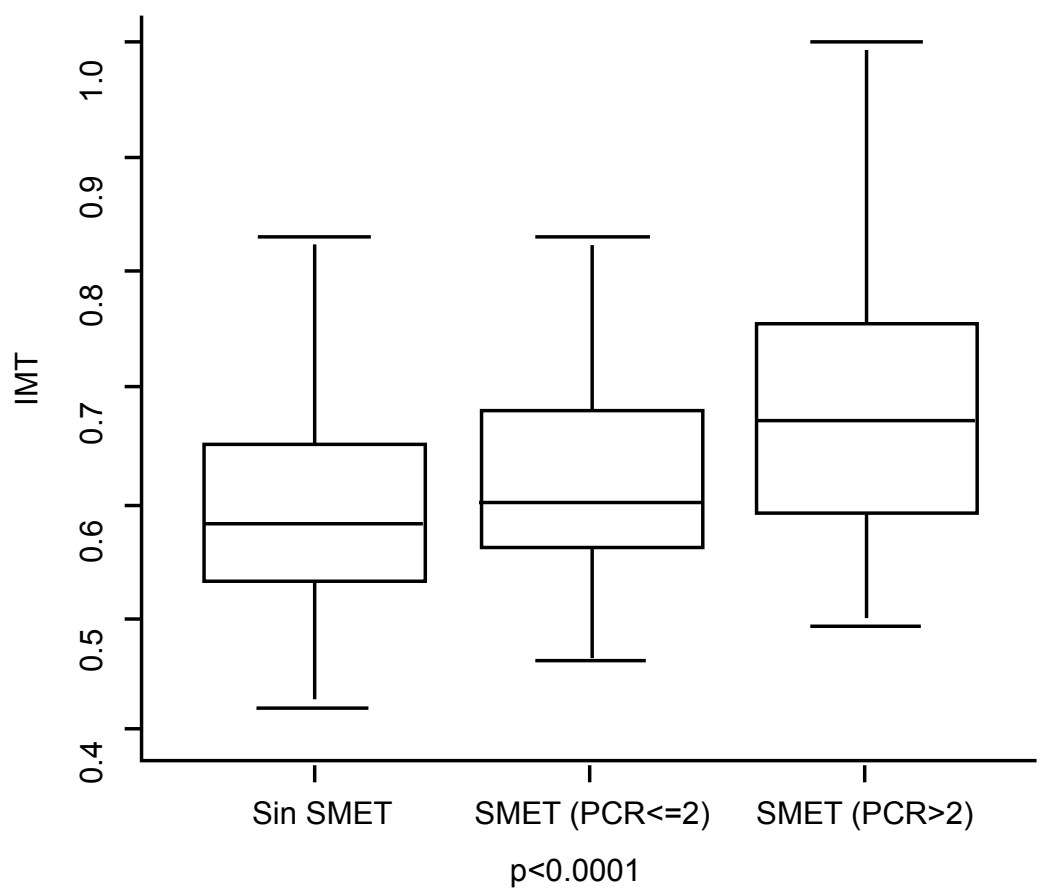

Figura 3: Grosor Intima-Media Carotídeo en Relación a Síndrome Metabólico y Proteína C-Reactiva ( $\mathrm{N}=944$ pacientes con EPCR <=10 mg/L) (Gráfico Box \& Whisker: la línea central representa la mediana, la caja el rango intercuartil y los extremos las observaciones mínima y máxima. 


\section{Resultados}

Se estudiaron 1.091 sujetos, de los cuales 999 se incluyeron en el análisis estadístico (508 hombres y 491 mujeres). Se excluyeron 81 sujetos con enfermedad aterosclerótica previa y 11 sujetos con antecedente de enfermedad inflamatoria. Así mismo, para el análisis de los sujetos con SMET, se incluyeron en el análisis sólo los que tenían ePCR $\leq 10 \mathrm{mg} / \mathrm{L}(\mathrm{n}=944)$.

La edad media del grupo fue $43.8 \pm 10.9$ años. La prevalencia de hipertensión fue de $30 \%$, dislipidemia $68 \%$, diabetes $12 \%$, tabaquismo activo $47 \%$ y $17 \%$ de los sujetos tenía antecedente familiar de cardiopatía coronaria. La prevalencia de SMET en esta muestra fue de $24 \%$. En la Tabla 1 se muestran las distintas prevalencias de factores de riesgo, como los valores de colesterol total, colesterol LDL, colesterol $\mathrm{HDL}$, triglicéridos, y EPCR, según sexo. Los valores de ePCR fueron significativamente mayores en las mujeres $(p<0.0001)$.

EI CIMT medio promedio de la muestra fue $0.61 \pm 0.1 \mathrm{~mm}$. En hombres, el CIMT medio fue de $0.62 \pm 0.1 \mathrm{~mm}$ y en mujeres de $0.60 \pm 0.1 \mathrm{~mm}$. Se detectó presencia de placa aterosclerótica en el $13.7 \%$ de los sujetos, sin diferencias por sexo. En la tabla 2 se muestran los valores promedio de CIMT según edad (en intervalos cada 10 años), y según sexo. El CIMT se correlacionó en forma directa y significativa a la edad, IMC, cintura, PA sistólica y diastólica, colesterol total, LDL, EPCR, y triglicéridos, e inversamente con el colesterol HDL (Tabla 3).

EI CIMT aumentó en forma significativa según el número de factores de riesgo. Los factores de riesgo que se consideraron fueron: edad $>45$ en hombres y $>55$ años en mujeres, presencia de diabetes, hipertensión arterial, colesterol total $>200 \mathrm{mg} / \mathrm{L}$, colesterol $\mathrm{HDL}<40$ en hombres y $<50 \mathrm{mg} / \mathrm{L}$ en mujeres, tabaquismo activo e historia familiar de cardiopatía coronaria (familiar directo hombre $<55$ y/o mujer $<65$ años). EI CIMT medio promedio según el número de factores de riesgo fue: con 0 factor de riesgo $=0.55 \pm 0.08 \mathrm{~mm} ; 1-2$ factores de riesgo $=0.59$ $\pm 0.09 \mathrm{~mm}$, y en los con 3 o más factores $=0.67 \pm 0.12$ $\mathrm{mm}$, con un valor $\mathrm{p}<0.0001$ para la tendencia (ANOVA). En la figura 1 se graficó el cambio de CIMT según el número de factores de riesgo, pero usando mediana y rango intercuartil (Gráfico Box \& Whisker).

Así mismo, el CIMT aumentó en forma significativa según el puntaje de Framingham: sujetos con puntaje de Framingham entre $0-5 \%$ presentaron un CIMT promedio de $0.58 \pm 0.09 \mathrm{~mm}$; los con puntaje $>5-10 \%$, presentaron un CIMT de $0.66 \pm 0.11$; los con puntaje $>10-20 \%$ un CIMT de $0.68 \pm 0.11$, y finalmente, los con puntaje Framingham $>20 \%$ un CIMT de $0.76 \pm 0.14$ $\mathrm{mm}(\mathrm{p}<0.0001$, para la tendencia). En la Figura 2 se muestra la misma información usando medianas y rango intercuartil.

El cambio en el CIMT en la población según la presencia de SMET se observa en la figura 3 , expresado como mediana y rango intercuartil (Gráfico Box \& Whisker). En los sujetos sin SMET $(n=713)$, el CIMT medio promedio fue significativamente menor que en los sujetos con SMET $(0.60 \pm 0.1 \mathrm{~mm}$ versus $0.66 \pm 0.12 \mathrm{~mm}$, respectivamente, $p<0.0001)$. En la figura 3 se puede apreciar que cuando se dividieron los sujetos con SMET en aquellos con o sin inflamación subclínica determinada por ePCR $>2 \mathrm{y} \leq 2 \mathrm{mg} / \mathrm{L}$, se ve que el CIMT es significativamente mayor en los sujetos con SMET + ePCR > 2. Los valores de CIMT promedio en esos grupos fueron: $(S M E T+e P C R \leq 2)=0.63 \pm 0.10$ $\mathrm{mm}$, y en los (SMET +ePCR > 2) $=0.69 \pm 0.13 \mathrm{~mm}$. Tanto en mujeres como en hombres se dieron los mismos resultados, no encontrándose diferencias significativas por sexo en la relación SMET y CIMT.

En el modelo de regresión lineal múltiple, el CIMT se asoció en forma significativa y en forma conjunta con Puntaje de Framingham $(p<0.0001)$ y (SMET + ePCR $>2)(p=0.01 ; R 2=0.19$ para el modelo $)$.

La Tabla 4 muestra los riesgos crudos (odds ratio) de tener un CIMT elevado (superior a $0.67 \mathrm{~mm}$, percentil 75 de la muestra) en función de los distintos factores de riesgo considerados. En forma conjunta (multivariada), el CIMT elevado se asocia al puntaje de Framingham $(\mathrm{OR}=1.12 ; \mathrm{IC}=1.08-1.15)$ y SMET + ePCR > 2 (OR = 1.76; IC = 1.10-2.82).

\section{Discusión}

En este estudio, en sujetos chilenos, hemos demostrado que el CIMT aumenta según la carga de factores de riesgo CV tradicionales y el puntaje de 
Framingham. Así mismo, el CIMT aumenta en forma significativa en los sujetos que presentan SMET, y cuando el SMET está presente y se asocia a niveles elevados de EPCR (inflamación subclínica), el CIMT es aún mayor, sugiriendo un aumento del riesgo aterosclerótico. Este estudio confirma la utilidad del CIMT para cuantificar carga de factores de riesgo $\mathrm{CV}$ en nuestro medio.

Numerosos estudios han confirmado que el CIMT aumenta según el número de factores de riesgo2-5, sin embargo, éste es el primer estudio en nuestro país que lo reporta. La importancia de estos resultados radica en que la medición de CIMT es un marcador de aterosclerosis subclínica, y por lo tanto, ayudaría a categorizar mejor el riesgo CV de cada individuo, sobretodo en sujetos en riesgo intermedio, como lo ha sugerido la Sociedad Americana de Cardiología1. Recientemente, fueron publicados los valores normales de CIMT en distintas ciudades latinoamericanas 27 , incluyendo Santiago, y quedó claro con ese reporte, que los valores de CIMT son sustancialmente distintos según la región estudiada. Por ello, es importante lo que se reporta en este estudio, que si bien los valores de CIMT en chilenos son menores en términos absolutos a los de estudios en USA o Europa, el CIMT en nuestro país se asocia a los factores clásicos de riesgo CV, como son la edad, el IMC, el colesterol total, HDL, LDL y triglicéridos, en forma semejante al de esas otras regiones americanas o europeas. Estos resultados apoyarían el uso de CIMT por los clínicos en forma rutinaria.

Así mismo, se demostró que el CIMT aumenta según aumenta el riesgo medido por el puntaje de Framingham. Si bien es cierto, esto podría parecer obvio, no lo es. En este estudio nosotros usamos las ecuaciones de Framingham publicadas por Wilson y cols. en $1998^{23}$, y que incluyen la diabetes como un factor más. La diferencia entre este puntaje versus la suma cruda de los factores, como se hizo primariamente (Figura 1), es que en el puntaje global de Framingham se realiza una categorización de cada variable, incluyendo la intensidad de alteración de cada factor de riesgo. La importancia de que el CIMT aumente según el puntaje de Framingham queda demostrado en nuestros resultados por el riesgo (odds) de Framingham de 1.13 para CIMT elevado: esto significa que por cada unidad de puntaje de
Framingham, el riesgo de tener CIMT elevado (> percentil 75) aumenta aproximadamente un $13 \%$ por cada punto adicional de Framingham, o sea es muy alto. Es decir, un puntaje de Framingham entre $10 \%$ y $20 \%$, que corresponde a un riesgo intermedio, casi duplicaría el riesgo de aterosclerosis subclínica en nuestra población santiaguina. Esto tiene importancia por 2 razones: a) porque a pesar de que el puntaje de Framingham es muy usado para estimar el riesgo CV, la evidencia sugiere que su capacidad predictiva es más bien baja, especialmente en los grupos con riesgo $(<10 \%)$, lo que apoyaría el uso de CIMT en esos pacientes, y 2) porque hoy ha sido demostrado que el CIMT es un buen predictor de eventos $\mathrm{CV}$, y sobretodo cerebrovasculares, como lo demostró recientemente el estudio MESA (Multi-Ethnic Study of Atherosclerosis) 28. Esta estrecha asociación entre CIMT y riesgo de Framingham ha sido también demostrada, y muy recientemente, por Karim y cols 29 , pero en un número menor de sujetos.

Uno de los objetivos primarios de nuestro estudio era determinar la asociación entre presencia de SMET y aterosclerosis subclínica. Los cardiólogos hemos sido ampliamente criticados por insistir en el Ilamado riesgo cardiometabólico, ya que según muchos investigadores, el SMET es sólo la suma de factores de riesgo individuales, y, por lo tanto, no tendría sentido hablar del mismo. Numerosos estudios, han demostrado que el síndrome como tal, confiere más riesgo $\mathrm{CV}$ que la suma de los factores individuales. Los estudios de Isommaa y cols. ${ }^{12}$, Lakka y cols. ${ }^{13}$ y otros ${ }^{14}$, así lo han demostrado. Nuestros resultados, en cierto modo apoyarían esta última aseveración: el CIMT fue significatimente mayor en los sujetos con SMET versus los sin SMET en población santiaguina. $Y$ más aún, cuando estos sujetos con SMET además presentaban EPCR elevada, el CIMT era mucho mayor. ¿Por qué, desde el punto de vista clínico, creemos que esto podría ser importante? EI SMET, aún, no es considerado una condición de riesgo o "enfermedad" por muchos clínicos. Muchos sujetos que califican para SMET sólo tienen una disglicemia leve, obesidad abdominal e hipertrigliceridemia, que en sentido estricto, no los califica con ningún factor de riesgo clásico que les confiera mayor riesgo CV. Sin embargo, el hallazgo de un CIMT más elevado, es decir, riesgo de 
aterosclerosis subclínica, podría llevarnos a ser más agresivos en la intervención de estos pacientes. Desde el punto de vista de riesgo CV futuro, independiente de la edad, el tener SMET, o SMET + EPCR alta, confirió, en las personas de este estudio, un riesgo de casi 2 veces de tener un CIMT elevado. Con respecto al riesgo que conferiría la EPCR en este contexto, es importante considerar que ya, por sí sola, la EPCR elevada da mayor riesgo, no sólo de eventos $\mathrm{CV}$, como ha sido demostrado por Ridker y cols. ${ }^{17}, 18$, sino que también de mayor aterosclerosis subclínica30 y diabetes ${ }^{11,20 .}$ El efecto potenciador de CIMT elevado en sujetos con SMET e inflamación, debiera ser investigado a futuro. Páramo y cols. 31 investigaron la relación de CIMT y distintos marcadores inflamatorios, incluyendo EPCR, pero encontraron una débil asociación con EPCR, a diferencia de nuestros resultados. Estos autores, además, no reportaron sobre la asociación SMET e inflamación. Chen y cols. ${ }^{30}$ publicaron sobre la relación de SMET, CIMT y EPCR, pero demostraron que el SMET sólo se asociaba a CIMT en mujeres, y la EPCR sólo confería más riesgo de placa carotídea en los hombres. En este sentido, el presente trabajo agrega información sobre la relación entre aterosclerosis subclínica, inflamación y SMET.

Finalmente, queremos destacar que nuestros resultados no pretenden subestimar la tremenda utilidad del uso del puntaje de Framingham para estimación del riesgo $\mathrm{CV}$, particularmente, cuando se evalúan poblaciones. Sólo queremos poner énfasis en las nuevas herramientas disponibles para la detección de aterosclerosis precoz, para lograr así una intervención individual más oportuna en los sujetos de riesgo intermedio o en aquellos de bajo riesgo, pero que pudieran estar subestimados por este puntaje. Así mismo, queremos destacar la importancia de la intervención precoz sobre la epidemia de SMET en nuestro país, que afecta no sólo a los adultos sino también a nuestros niños 32 .

\section{Limitaciones}

Nuestro estudio tiene limitaciones. Primero, corresponde a un estudio transversal, donde no se puede hablar de causalidad. La población estudiada es relativamente joven, sin representación de adultos $>70$ años. Por otra parte, no se realizó ajuste por uso de medicamentos. Si bien estos fármacos pudieran haber influido en la relación de CIMT con los factores de riesgo, el SMET y la EPCR, lo más probable es que hubieran atenuado las relaciones encontradas, de manera que no invalidan los resultados. Finalmente, para demostrar si el riesgo aterosclerótico de eventos $\mathrm{CV}$, determinado por el CIMT, aumenta más en aquellos sujetos con más factores de riesgo, o con SMET e inflamación, se necesita seguir a estos sujetos en forma prospectiva para medir eventos $\mathrm{CV}$ duros.

\section{Referencias}

1. SMITH SC JR, GREENLAND P, GRUNDY SM. AHA Conference Proceedings. Prevention conference $V$ : Beyond secondary prevention: Identifying the high-risk patient for primary prevention: executive summary. American Heart Association. Circulation 2000; 101: 111-116.

2. CHAMBLESS LE, HEISS G, FOLSOM AR, ROSAMOND W, SZKLO M, SHARRETT AR, et al. Association of coronary heart disease incidence with carotid arterial wall thickness and major risk factors: the Atherosclerosis Risk in Communities (ARIC) Study, 1987-1993. Am J Epidemiol 1997; 146: 483-494.

3. MANNAMI T, KONISHI M, BABA $S, N I S H I N$, TERAO A. Prevalence of asymptomatic carotid atherosclerotic lesions detected by high-resolution ultrasonography and its relation to cardiovascular risk factors in the general population of a Japanese city: the Suita study. Stroke 1997; 28: 518-525.
4. O'LEARY DH, POLAK JF, KRONMAL RA, KITTNER SJ, BOND MG, WOLFSON SK JR, et al. Distribution and correlates of sonographically detected carotid artery disease in the Cardiovascular Health Study. The CHS Collaborative Research Group. Stroke 1992; 23: 1752-1760.

5. POLI A, TREMOLI E, COLOMBO A, SIRTORI M, PIGNOLI P, PAOLETTI R. Ultrasonographic measurement of the common carotid artery wall thickness in hypercholesterolemic patients. A new model for the quantitation and follow-up of preclinical atherosclerosis in living human subjects. Atherosclerosis 1988; 70: 253-261.

6. HODIS HN, MACK WJ, LABREE L, SELZER RH, LIU CR, LIU $\mathrm{CH}$, et al. The role of carotid arterial intima-media thickness in predicting clinical coronary events. Ann Intern Med 1998; 128: 262-269.

7. O'LEARY DH, POLAK JF, KRONMAL RA, MANOLIO TA, BURKE GL, WOLFSON SK JR. Carotid-artery intima and 
media thickness as a risk factor for myocardial infarction and stroke in older adults. Cardiovascular Health Study Collaborative Research Group. N Engl J Med 1999; 340: 14-22.

8. LORENZ MW, MARKUS HS, BOTS ML, ROSVALL M, SITZER M. Prediction of clinical cardiovascular events with carotid intima-media thickness: a systematic review and meta-analysis. Circulation 2007; 115: 459-467.

9. ALBERTI KG, ZIMMET P, SHAW J. The metabolic syndrome-a new worldwide definition. Lancet 2005; 366: 1059-1062.

10. ECKEL RH, GRUNDY SM, ZIMMET PZ. The metabolic syndrome. Lancet 2005; 365: 1415-1428.

11. HAFFNER SM. The metabolic syndrome: inflammation, diabetes mellitus, and cardiovascular disease. Am J Cardiol 2006; 97: 3A-11A.

12. ISOMAA B, ALMGREN P, TUOMI T, FORSEN B, LAHTI K, NISSEN M, et al. Cardiovascular morbidity and mortality associated with the metabolic syndrome. Diabetes Care 2001; 24: 683-689.

13. LAKKA HM, LAAKSONEN DE, LAKKA TA, NISKANEN LK, KUMPUSALO E, TUOMILEHTO J, et al. The metabolic syndrome and total and cardiovascular disease mortality in middle-aged men. JAMA 2002; 288: 2709-2716.

14. MALIK S, WONG ND, FRANKLIN SS, KAMATH TV, L'ITALIEN GJ, PIO JR, et al. Impact of the metabolic syndrome on mortality from coronary heart disease, cardiovascular disease, and all causes in United States adults. Circulation 2004; 110: 1245-1250.

15. IGLSEDER B, CIP P, MALAIMARE L, LADURNER G, PAULWEBER $B$. The metabolic syndrome is a stronger risk factor for early carotid atherosclerosis in women than in men. Stroke 2005; 36: 1212-1217.

16. SIPILA K, MOILANEN L, NIEMINEN T, REUNANEN A, JULA A, SALOMAA $V$, et al. Metabolic syndrome and carotid intima media thickness in the Health 2000 Survey. Atherosclerosis 2009; 204: 276-81.

17. RIDKER PM, CUSHMAN M, STAMPFER MJ, TRACY RP, HENNEKENS $\mathrm{CH}$. Inflammation, aspirin, and the risk of cardiovascular disease in apparently healthy men. N Engl J Med 1997; 336: 973-979.

18. RIDKER PM, BURING JE, COOK NR, RIFAI N. C-reactive protein, the metabolic syndrome, and risk of incident cardiovascular events: an 8-year follow-up of 14719 initially healthy American women. Circulation 2003; 107: 391-397.

19. DANESH J, WHEELER JG, HIRSCHFIELD GM, EDA S, EIRIKSDOTTIR G, RUMLEY A, et al. C-reactive protein and other circulating markers of inflammation in the prediction of coronary heart disease. N Engl J Med 2004; 350: 1387-1397.

20. HANLEY AJ, FESTAA, D'AGOSTINO RB JR, WAGENKNECHT LE, SAVAGE PJ, TRACY RP, et al. Metabolic and inflammation variable clusters and prediction of type 2 diabetes: factor analysis using directly measured insulin sensitivity. Diabetes 2004; 53: 1773-1781.
21. SATTAR N, GAW A, SCHERBAKOVA O, FORD I, O'REILLY DS, HAFFNER SM, et al. Metabolic syndrome with and without C-reactive protein as a predictor of coronary heart disease and diabetes in the West of Scotland Coronary Prevention Study. Circulation 2003; 108: 414-419.

22. Executive Summary of The Third Report of The National Cholesterol Education Program (NCEP) Expert Panel on Detection, Evaluation, And Treatment of High Blood Cholesterol In Adults (Adult Treatment Panel III). JAMA 2001; 285: 2486-2497.

23. WILSON PW, D'AGOSTINO RB, LEVY D, BELANGER AM, SILBERSHATZ H, KANNEL WB. Prediction of coronary heart disease using risk factor categories. Circulation 1998; 97: 1837-1847.

24. PEARSON TA, BROWN WV, DONATO K, FRANKLIN FA, JR., LUEPKER RB, MCBRIDE PE, et al. Lipids. Workshop IV. AHA Prevention Conference III. Behavior change and compliance: keys to improving cardiovascular health. Circulation 1993; 88: 1397-1401.

25. TOUBOUL PJ, HENNERICI MG, MEAIRS S, ADAMS $\mathrm{H}$, AMARENCO P, DESVARIEUX M, et al. Mannheim intimamedia thickness consensus. Cerebrovasc Dis 2004; 18: 346-349.

26. TOUBOUL PJ, HENNERICI MG, MEAIRS S, ADAMS $\mathrm{H}$, AMARENCO P, BORNSTEIN N, et al. Mannheim carotid intima-media thickness consensus (2004-2006). An update on behalf of the Advisory Board of the 3rd and 4th Watching the Risk Symposium, 13th and 15th European Stroke Conferences, Mannheim, Germany, 2004, and Brussels, Belgium, 2006. Cerebrovasc Dis 2007; 23: 75-80.

27. SCHARGRODSKY H, HERNANDEZ-HERNANDEZ R, CHAMPAGNE BM, SILVA $H$, VINUEZA R, SILVA AYCAGUER LC, et al. CARMELA: assessment of cardiovascular risk in seven Latin American cities. Am J Med 2008; 121: 58-65.

28. FOLSOM AR, KRONMAL RA, DETRANO RC, O'LEARY DH, BILD DE, BLUEMKE DA, et al. Coronary artery calcification compared with carotid intima-media thickness in the prediction of cardiovascular disease incidence: the Multi-Ethnic Study of Atherosclerosis (MESA). Arch Intern Med 2008; 168: 1333-1339.

29. KARIM R, HODIS HN, DETRANO R, LIU CR, LIU CH, MACK WJ. Relation of framingham risk score to subclinical atherosclerosis evaluated across three arterial sites. Am J Cardiol 2008; 102: 825-830.

30. CHEN PC, CHIEN KL, HSU HC, SU TC, CHANG CW, SUNG FC, et al. C-reactive protein and the metabolic syndrome correlate differently with carotid atherosclerosis between men and women in a Taiwanese community. Metabolism 2008; 57: 1023-1028.

31. PARAMO JA, ORBE J, BELOQUI O, COLINA I, BENITO A, RODRIGUEZ JA, et al. Association of age, inflammatory markers and subclinical atherosclerosis in subjects free from cardiovascular disease. Med Clin (Barc) 2008; 131: 361-366. 
M. Acevedo, P. Arnaíz, R. Corbalán, I. Godoy, D. Morales, M. Chalhub, et al.

32. BURROWS R, BURGUENO M, LEIVA L, CEBALLOS X, GUILLIER I, GATTAS V, et al. Cardiovascular risk and metabolic profile in obese children and adolescents with low insulin sensitivity. Rev Med Chil 2005; 133: 795-804. 